Physics or Astrophysics/Header

\title{
The VKS experiment: turbulent dynamical dynamos
}

\author{
VKS Collaboration : Sébastien Aumaître ${ }^{\text {a }}$ \\ Michael Berhanu ${ }^{\mathrm{b}}$ Mickael Bourgoin ${ }^{\mathrm{c}}$ Arnaud Chiffaudel ${ }^{\mathrm{a}}$ \\ François Daviaud a Bérengère Dubrulle ${ }^{\mathrm{a}}$ Stephan Fauve ${ }^{\mathrm{b}}$ \\ Louis Marié ${ }^{a}$ Romain Monchaux ${ }^{a}$ Nicolas Mordant ${ }^{b}$ \\ Philippe Odier ${ }^{\mathrm{c}}$ François Pétrélis ${ }^{\mathrm{b}}$ Jean-François Pinton ${ }^{\mathrm{c}}$ \\ Nicolas Plihon ${ }^{\mathrm{c}}$ Florent Ravelet ${ }^{\mathrm{a}}$ Romain Volk ${ }^{\mathrm{c}}$ \\ a Service de Physique de l'Etat Condensé, CNRS \& CEA Saclay, \\ F-91191 Gif-sur-Yvette, France \\ b Laboratoire de Physique Statistique, CNRS \&ु École Normale Supérieure, \\ 24 rue Lhomond, F-75005 Paris, France \\ c Laboratoire de Physique de l'École Normale Supérieure de Lyon, \\ CNRS \& Université de Lyon, F-69364 Lyon, France \\ Received $* * * * *$; accepted after revision +++++
}

\begin{abstract}
The VKS experiment studies dynamo action in the flow generated inside a cylinder filled with liquid sodium by the rotation of coaxial impellers (the von Kármán geometry). We report observations related to the self-generation of a stationary dynamo when the flow forcing is symmetric, i.e. when the impellers rotate in opposite directions at equal angular velocities. The bifurcation is found to be supercritical, with a neutral mode whose geometry is predominantly axisymmetric. We then report the different dynamical dynamo regimes observed when the flow forcing is asymmetric, including magnetic field reversals. We finally show that these dynamics display characteristic features of low dimensional dynamical systems despite the high degree of turbulence in the flow.
\end{abstract}

\section{L'expérience VKS : dynamiques d'une dynamo turbulente}

\section{Résumé}

L'expérience VKS étudie l'effet dynamo dans l'écoulement de von Kármán engendrée dans un cylindre par la contre-rotation des deux turbines coaxiales. Nous décrivons d'abord l'apparition et l'auto-entretien d'une dynamo statistiquement stationnaire, engendrée quand les turbines sont en contra-rotation exacte. L'in- 
stabilité dynamo se développe au travers d'une bifurcation super-critique avec un mode neutre essentiellement axisymétrique. Nous discutons ensuite l'observation de régimes dynamique riches, engendrés lorsque les turbines sont en contra-rotation à des vitesses différentes, c'est-à-dire en présence d'une rotation globale de l'écoulement. Renversements erratiques, oscillations et régimes de bouffées intenses de champ magnétique sont alors observés. Nous montrons que ces comportements, engendrés pour des valeurs voisines des paramètres de contrôle de l'écoulement, ont des caractéristiques très similaires aux systèmes chaotiques de faible dimensionalité en dépit de la turbulence importante de l'écoulement.

Key words: Dynamo; Magnetohydrodynamics ; Turbulence

Mots-clés : Dynamo; Magnétohydrodynamique; Turbulence

\section{Introduction}

The generation of electricity from mechanical work has been one of the main achievements of physics by the end of the XIX ${ }^{\text {th }}$ century. In 1919, Larmor proposed that a similar process can generate the magnetic field of the sun from the motion of an electrically conducting fluid. However, fluid dynamos are more complex than industrial ones and it is not easy to find laminar flow configurations that generate magnetic fields [1]. Two simple but clever examples have been found in the seventies [2] and have led more recently to successful experiments [3]. These experiments have shown that the observed thresholds are in good agreement with theoretical predictions [4] made by considering only the mean flow, whereas the saturation level of the magnetic field cannot be described with a laminar flow model (without using an ad-hoc turbulent viscosity). [5]. These observations have raised many questions: what happens for flows without geometrical constraints such that fluctuations are of the same order of magnitude as the mean flow? Is the dynamo threshold strongly increased due to the lack of coherence of the driving flow $[6,7]$ or does the prediction based on the mean flow still give a reasonable order of magnitude [8]? What is the nature of the dynamo bifurcation in the presence of large velocity fluctuations? All these questions, and others motivated by geophysical or astrophysical dynamos [9], have led several teams to try to generate dynamos in flows with a high level of turbulence $[10,11]$.

We report here the dynamo regimes observed in the von Kármán Sodium (VKS) series of experiments made in 2006-2007 [12,13,14]. 


\section{Experimental setup}

A sketch of the experiment is shown in figure 1. The flow is generated by rotating two disks of radius $154.5 \mathrm{~mm}, 371 \mathrm{~mm}$ apart in a cylindrical vessel, $2 R=412 \mathrm{~mm}$ in inner diameter and $524 \mathrm{~mm}$ in length. The disks are fitted with 8 curved blades of height $h=41.2 \mathrm{~mm}$. These impellers are driven at rotation frequencies $\left(F_{1}, F_{2}\right)$ up to $26 \mathrm{~Hz}$ by $300 \mathrm{~kW}$ available motor power. An oil circulation in the outer copper cylinder maintains a regulated temperature in the range $110-160^{\circ} \mathrm{C}$. The mean flow has the following characteristics: the fluid is ejected radially outward by the disks; this drives an axial flow toward the disks along their axis and a recirculation in the opposite direction along the cylinder lateral boundary. In addition, in the case of counter-rotating disks studied here, the presence of a strong axial shear of azimuthal velocity in the mid-plane between the impellers generates a high level of turbulent fluctuations $[15,16]$. The kinetic Reynolds number is $R e=K R^{2} F_{i} / \nu$, where $\nu$ is the kinematic viscosity and $K=0.6$ is a coefficient that measures the efficiency of the impellers [16]. Re can be increased up to $5 \times 10^{6}$ : the corresponding magnetic Reynolds number is $R_{m}=K \mu_{0} \sigma R^{2} F_{i} \approx 49$ (at $120^{\circ} \mathrm{C}$ ), where $\mu_{0}$ is the magnetic permeability of vacuum and $\sigma$ is the electrical conductivity of sodium.
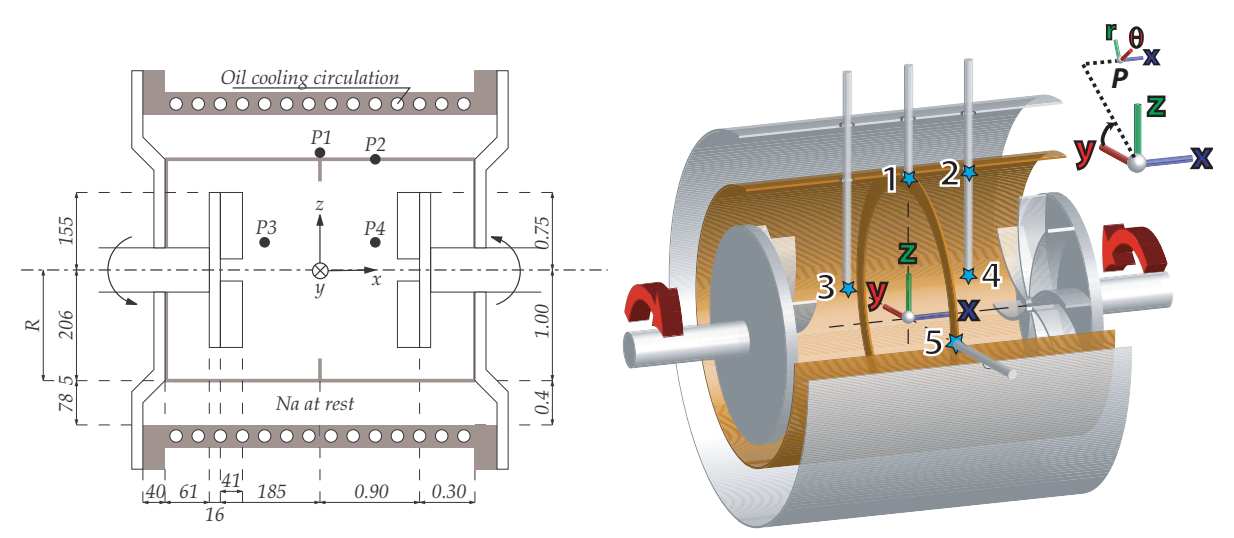

Figure 1. Experimental setup. Note the curved impellers, the inner cylinder which separates the flow volume from the blanket of surrounding sodium and the thin annulus in the mid plane. Also shown are the holes through which the 3D Hall probes are inserted into the vessel for magnetic measurements. Location in $(r / R, \theta, x / R)$ coordinates of available measurement points: $P_{1}(1, \pi / 2,0)$; $P_{2}(1, \pi / 2,0.58) ; P_{3}(0.25, \pi / 2,-0.58) ; P_{4}(0.25, \pi / 2,0.58) ; P_{5}(1, \pi, 0)$ (see table 1 for details concerning available measurements in different experimental runs). When referring to the coordinates of magnetic field vector $\vec{B}$ measured in the experiment at these different points, we will use either the cartesian projection $\left(B_{x}, B_{y}, B_{x}\right)$ on the frame $(\vec{x}, \vec{y}, \vec{z})$ or the cylindrical projection $\left(B_{r}, B_{\theta}, B_{x}\right)$ on the frame $(r, \theta, x)$ $\left(B_{\theta}=-B_{y}\right.$ and $B_{r}=B_{z}$ for measurements at points $P 1, P 2$ and $P 3$.) 
Table 1

\begin{tabular}{|c|c|c|c|c|c|c|c|}
\hline Runs & Impellers & Dynamo & P1 & P2 & P3 & P4 & P5 \\
\hline \hline VKS2f & stainless steel & No & MP & - & - & - & - \\
VKS2g & iron & Yes & MP & G & - & - & - \\
VKS2h & iron & Yes & G & - & - & - & MP \\
VKS2i & iron & Yes & - & - & MP & G & - \\
\hline
\end{tabular}

Experimental configuration of the four successive runs discussed here. VKS2g (september 2006) is the first run with dynamo action. Labels $M P$ or $G$ indicate whether a 3D probe array of Hall sensors or a single 3D gaussmeter was used. P\# (cf figure 1) is the location of the measurement - the inner-most sensor for the probe array.

Compared to previous VKS experiments, three modifications have been performed regarding flow generation and boundary conditions. A first modification consists of surrounding the flow by sodium at rest in another concentric cylindrical vessel, $578 \mathrm{~mm}$ in inner diameter. This has been shown to decrease the dynamo threshold in kinematic computations based on the mean flow velocity [16]. The total volume of liquid sodium is $150 \mathrm{l}$. A second geometrical modification consists of attaching an annulus of inner radius $175 \mathrm{~mm}$ and thickness $5 \mathrm{~mm}$ along the inner cylinder in the mid-plane between the disks. Water experiments have shown that its effect on the mean flow is to make the shear layer sharper around the mid-plane. In addition, it reduces low frequency turbulent fluctuations, but the rms velocity fluctuations are almost unchanged (of order $40-50 \%$ ) [17]. Finally, the impellers driving the flow are made of soft iron. Using boundary conditions with a high permeability in order to change the dynamo threshold has been already proposed [18]. It has been also shown that in the case of a Ponomarenko or G. O. Roberts flows, the addition of an external wall of high permeability can decrease the dynamo threshold [19]. Kinematic simulations of the VKS mean flow have shown that different ways of taking into account the sodium behind the disks lead to an increase of the dynamo threshold ranging from $12 \%$ to $150 \%$ [20]. Other kinematic simulations using a model flow show that the threshold with boundaries of high magnetic permeability is lower than the one obtained by replacing the sodium behinf the disks by an insultating medium [21], although the actual behavior may be more complex.

Magnetic measurements are performed using 3D Hall probes, and recorded with a National Instruments PXI digitizer. We use both a single-point (three components) Bell probe (hereafter label $G$-probe) connected to its associated gaussmeter and a custom-made array where the 3D magnetic field is sampled at 10 locations along a line (hereafter called $S M$-array), every $28 \mathrm{~mm}$. The array is made from Sentron 2SA-1M Hall sensors, and is air-cooled to keep the sensors temperature between 35 and $45^{\circ} \mathrm{C}$. For both probes, the dynamical 
range is $70 \mathrm{~dB}$, with an $\mathrm{AC}$ cut-off at $400 \mathrm{~Hz}$ for the gaussmeter and $1 \mathrm{kHz}$ for the custom-made array; signals have been sampled at rates between 1 and $5 \mathrm{kHz}$. The locations of the probe in each of the experimental runs discussed here are reported in table I.

3 Dynamo generation, symmetric forcing, $F_{1}=F_{2}$

We first consider the behavior when the flow is forced by impellers counterrotating at equal rates, $F_{1}=F_{2}$. Figure 2 shows the time recording of the three components of $\vec{B}$ when $R_{m}$ is increased from 19 to 40. The largest component, $B_{y}$, is tangent to the cylinder at the measurement location. It increases from a mean value comparable to the Earth magnetic field to roughly $40 \mathrm{G}$. The mean values of the other components $B_{x}$ and $B_{z}$ also increase (not visible on the figure because of fluctuations). Both signs of the components have been observed in different runs, depending on the sign of the residual magnetization of the disks. All components display strong fluctuations as could be expected in flows with Reynolds numbers larger than $10^{6}$.

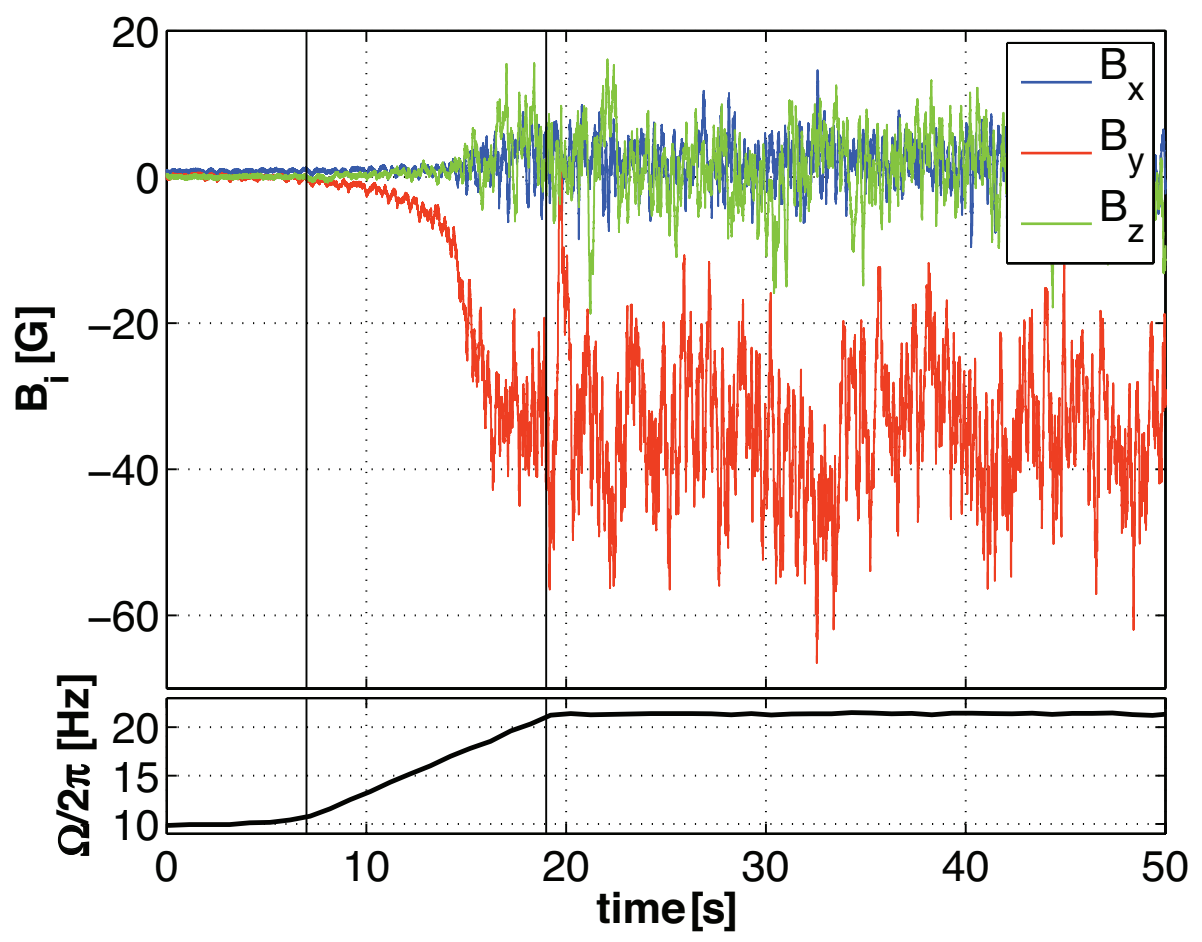

Figure 2. Three component of the magnetic field generated by dynamo action at $F_{1}=F_{2}$ measured at point $P 1$ in the experiment VKS2g.

Figure 3a shows the mean values of the components $\left\langle B_{i}\right\rangle$ of the magnetic field and Fig. $3 \mathrm{~b}$ their fluctuations $B_{i r m s}$ versus $R_{m}$. The fluctuations are all in the same range ( $3 \mathrm{G}$ to $8 \mathrm{G}$, at $30 \%$ above threshold) although the corre- 
sponding mean values are very different. The time average of the square of the total magnetic field, $\left\langle\vec{B}^{2}\right\rangle$, is displayed in the inset of Fig. 3a. No hysteresis is observed. Linear fits of $\left\langle B_{y}\right\rangle$ or $B_{\text {irms }}$ displayed in Fig. 3 define a critical magnetic Reynolds number $R_{m}^{c} \sim 31$ whereas the linear fit of $\left\langle\vec{B}^{2}\right\rangle$ gives a larger value $R_{m}^{0} \sim 35$. The latter is the one that should be considered in the case of a supercritical pitchfork bifurcation. The rounding observed close to threshold could then be ascribed to the imperfection due to the ambient magnetic field (Earth field, residual magnetization of the disks and other magnetic perturbations of the set-up). The actual behavior may be more complex because this bifurcation takes place on a strongly turbulent flow, a situation for which no rigorous theory exists. The inset of Fig. $3 \mathrm{~b}$ shows that the variance $B_{r m s}^{2}=\left\langle(\vec{B}-\langle\vec{B}\rangle)^{2}\right\rangle$ is not proportional to $\left\langle B^{2}\right\rangle$. Below the dynamo threshold, the effect of induction due to the ambient magnetic field is observed. $B_{r m s} /\left\langle B^{2}\right\rangle^{1 / 2}$ first behaves linearly at low $R_{m}$, but then increases faster as $R_{m}$ becomes closer to the bifurcation threshold. We thus show that this seems to be a good quantity to look at as a precursor of a dynamo regime. In addition, we observe that it displays a discontinuity in slope in the vicinity of $R_{m}^{c}$ in an analogous way of some response functions at phase transitions or bifurcations in the presence of noise. Note however that the shape of the curves depends on the measurement point and they cannot be superimposed with a scaling factor as done for $\left\langle B^{2}\right\rangle$ versus $R_{m}$ in the inset of Fig. 3a. The probability density functions (PDF) of the fluctuations of the three components of the induced magnetic field (not displayed) are roughly gaussian. The PDFs of fluctuations below threshold, i.e., due to the induction resulting from the ambient magnetic field, are similar to the ones observed in the self-generating regime. We do not observe any non gaussian behavior close to threshold which would result from an on-off intermittency mechanism [22]. Possible reasons are the low level of small frequency velocity fluctuations [23] or the imperfection of the bifurcation that results from the ambient magnetic field [24].

Dissipated power by Ohmic losses is another important characterization of dynamo action. Indication obtained from the commercial indicator on the electric power source of the motors show that, $30 \%$ above threshold, there is an excess power consumption of $15-20 \%$ with respect to a flow driving power of the order of $100 \mathrm{~kW}$.

The effect of iron disks deserves additional discussion. Indeed, using stainless steel impellers instead of iron ones does not generate a magnetic field up to the maximum possible rotation frequency of the disks $(F=26 \mathrm{~Hz})$. In addition, the dynamo threshold during the first run was about $20 \%$ larger than in the next runs for which all the measurements were then perfectly reproducible. However, no effect of remanence that would lead to a hysteretic behavior close to the bifurcation threshold has been observed. As demagnetization of pure iron occurs for field amplitudes of the order of the Earth field, i.e., much smaller than the fields generated by the dynamo, the iron disks do not impose 

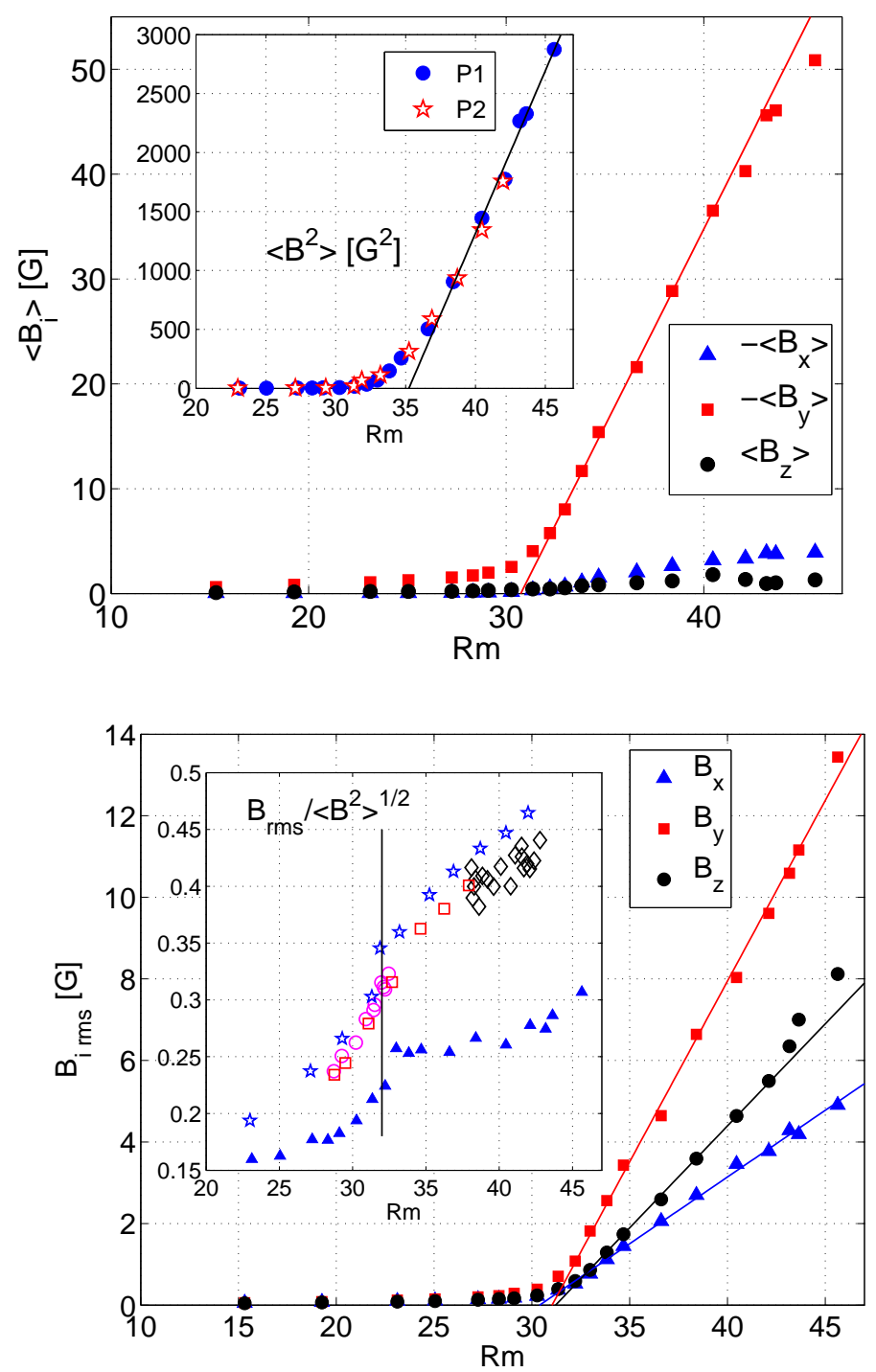

Figure 3. a) Mean values of the three components of the magnetic field recorded at $P 1$ versus $R_{m}\left(T=120^{\circ} \mathrm{C}\right):(\boldsymbol{\Lambda})-\left\langle B_{x}\right\rangle,(\mathbf{\square})-\left\langle B_{y}\right\rangle,(\bullet)\left\langle B_{z}\right\rangle$. The inset shows the time average of the square of the total magnetic field as a function of $R_{m}$, measured at $P 1(\bullet)$, or at P2 $(\star)$ after being divided by 1.8 . b) Standard deviation of the fluctuations of each components of the magnetic field recorded at $P 1$ versus $R_{m}$. The inset shows $B_{r m s} /\left\langle B^{2}\right\rangle^{1 / 2}$. Measurements done at $P 1$ : $(\boldsymbol{\Lambda}) T=120^{\circ} \mathrm{C}$, frequency increased up to $22 \mathrm{~Hz}$; Measurements done at $P 2:(\star) T=120^{\circ} \mathrm{C}$, frequency decreased from 22 to $16.5 \mathrm{~Hz},(\square) T=156^{\circ} \mathrm{C}$, frequency increased up to $22 \mathrm{~Hz}$, (o) $\Omega / 2 \pi=16.5 \mathrm{~Hz}, T$ varied from 154 to $116^{\circ} \mathrm{C},(\diamond) \Omega / 2 \pi=22 \mathrm{~Hz}, T$ varied from 119 to $156^{\circ} \mathrm{C}$. The vertical line corresponds to $R_{m}=32$.

any permanent magnetization but mostly change the boundary condition for the magnetic field generated in the bulk of the flow. This changes the dynamo threshold and the near critical behavior for amplitudes below the coercitive field of pure iron. It should be also emphasized that the axisymmetry of the 
set-up cannot lead to Herzenberg-type dynamos [25]. In addition, these rotor dynamos display a sharp increase of the field at threshold and their saturation is mostly limited by the available motor power [25]. On the contrary, we observe a continuous bifurcation with a saturated magnetic field in good agreement with a scaling law derived for a fluid dynamo.

No secondary instability is observed when $F$ is increased further up to its maximum value, $26 \mathrm{~Hz}$, and the dynamics as well as the mean field geometry are unchanged. The mean field is mostly azimuthal close to the flow periphery whereas the azimuthal and axial components are of the same order of magnitude in the bulk of the flow. The radial component is much weaker. These observations show that the mean magnetic field differs from the one computed numerically by taking into account the mean flow alone. It was found to be an equatorial dipole $[26,27,16]$, thus leading to a strong radial component in the mid-plane between the impellers. A simple model of an $\alpha-\omega$ dynamo has been proposed to understand the experimentally observed axial dipole [28,29]: it takes into account the helical nature of the velocity corresponding to the flow ejected by the centrifugal force close to each impeller between the blades. This gives an $\alpha$-effect localized close to the disks that transforms an azimuthal field into a poloidal one, whereas differential rotation converts also very efficiently the poloidal component into toroidal by $\omega$ effect. However, the distribution of helicity in three-dimensional turbulence remains a challenging question. Induction measurements in $[30,31]$ show another possible contribution to the alpha effect, from spatial inhomogeneities in the turbulence intensity. As calculated in [32], there is a contribution to the mean-field alpha tensor coming from the inhomogeneity of turbulent fluctuations, with resulting electromotive force $\epsilon \sim(\mathrm{g} \cdot \Omega) \mathbf{B}$, where $\Omega$ is the flow vorticity and $\mathbf{g}$ the normalized gradient of turbulent fluctuations, $\mathbf{g}=\left(\nabla u^{2}\right) / \overline{u^{2}}$. In this case again an azimuthal $B_{\theta}$ field generates a $j_{\theta}$ current, and therefore an axial magnetic field. Distinguishing between these mechanisms (which may be linked) will need further measurements, ideally from nearby velocity and magnetic probes.

We note further that, although non axisymmetric fluctuations are essential to explain the geometry of the mean magnetic field, they have a surprisingly weak effect on the large scale dynamics of the field. In particular, in the case of impellers counter-rotating at the same frequency, these fluctuations never induce a change of polarity of the mean magnetic field even close to the bifurcation threshold, although the magnetic energy density is much smaller than the one of the turbulent velocity fluctuations. 


\section{Dynamos for asymmetric forcing, $F_{1} \neq F_{2}$}

In the experiment, the rotation rates $\left(F_{1}, F_{2}\right)$ of the driving impellers can be independently adjusted and this gives an additional degree of freedom. Starting from a symmetric flow forcing, one can progressively change the rotation frequency of one disk and explore regimes in which the faster disk imposes some kind of global rotation to the flow, a feature common to most natural dynamos. We show in figure 4 an inspection of parameter space when the flow is driven with disks rotating at different speeds.

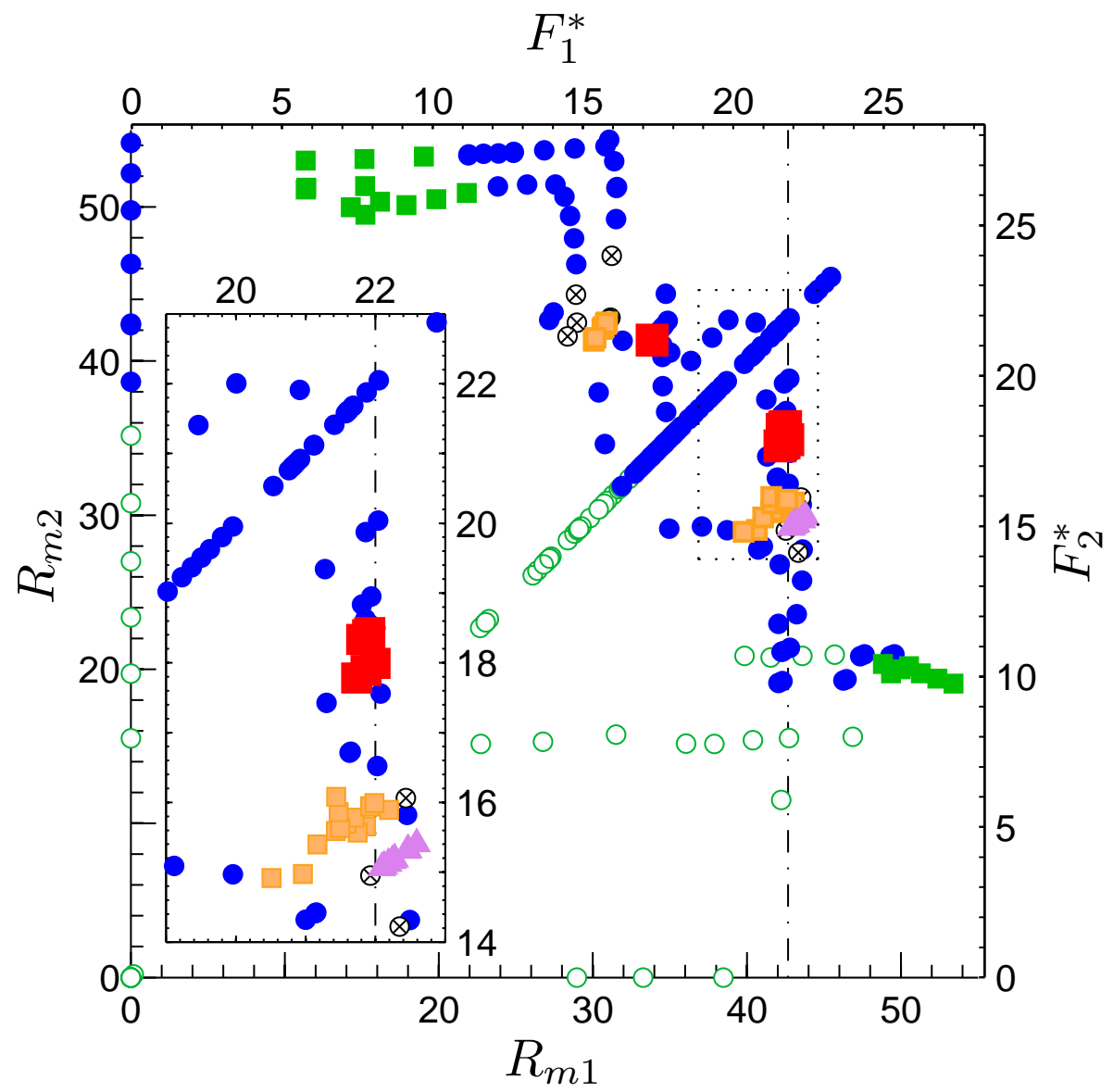

Figure 4. Observed dynamo regimes. The parameter space is labeled by the magnetic Reynolds numbers $R_{m i}$ and, for clarity, by the frequencies corrected for mean temperature variation: $F_{i}^{*}=\left[\sigma(T) / \sigma\left(120^{\circ} \mathrm{C}\right)\right] F_{i}$. Legend: no dynamo (green $\circ ; B \lesssim 10 \mathrm{G}$ for more than $180 \mathrm{~s}$ ), statistically stationary dynamos (blue •); oscillatory dynamos (green squares); limit cycles (red squares), magnetic reversals (orange squares), bursts (purple triangles) and transient magnetic extinctions $(\otimes)$. All regimes have been characterized from recodings spanning 3 minutes long.

As the difference in angular velocities is varied from the symmetric frocing $F_{1}=F_{2}$, we observe that dynamo action is preserved and that the magnetic field measured inside the flow or at its boundary becomes time-dependent. 
In a small parameter range, $\Delta F_{i} / F_{i} \sim 20 \%$, a variety of dynamical regimes, oscillations, intermittent bursts (not shown), as well as dynamos with random reversals are observed. We also find pockets of parameters for which we could not record the growth of a dynamo during 3-minute long runs -corresponding to over 3000 forcing time scales.

We describe further below the case of irregular reversals. For $\left(F_{1}=16 \mathrm{~Hz}, F_{2}=\right.$ $22 \mathrm{~Hz}$ ), a dynamical regime in which the magnetic field randomly changes its polarity is observed - cf. figure 5. All three components of the dynamo field switch polarity, so that $\mathbf{B}$ changes to $-\mathbf{B}$. For each polarity, the amplitude of the magnetic field has strong fluctuations, with an rms fluctuation level of the order of $20 \%$ of the mean. This level of fluctuation is due to the very intense turbulence of the flow, as the kinetic Reynolds number exceeds $10^{6}$. Reversals occur randomly and have been followed for up to 45 minutes, i.e. 54000 characteristic time scales of the flow forcing. Note however that the amplitude of the magnetic field, much larger than the Earth field, is the same for both polarities. Standard deviations are of the same order of magnitude as the mean values, although better statistics may be needed to fully converge these estimates. The mean duration of each reversal, $\tau \sim 5 \mathrm{~s}$, is longer than magnetohydrodynamics time scales: the flow integral time scale is of the order of the inverse of the rotation frequencies, i.e. $0.05 \mathrm{~s}$, and the ohmic diffusive time scale is roughly $\tau_{\eta} \sim 0.4 \mathrm{~s}$. In the regime reported in figure 5 , the polarities do not have the same probability of observation. Phases with a positive polarity for the largest magnetic field component have on average longer duration $\left(\left\langle T_{+}\right\rangle=120 \mathrm{~s}\right)$ than phases with the opposite polarity $\left(\left\langle T_{-}\right\rangle=50 \mathrm{~s}\right)$.

Concerning the dynamics of field reversals, a natural question is related to the connection between $\mathbf{B}$ and $-\mathbf{B}$ in time. The equations of magnetohydrodynamics are symmetric under the transformation so that the selection of a polarity is a broken symmetry at the dynamo bifurcation threshold. The sequences of opposite polarities displayed in figure 5 act as magnetic domains along the time axis, with Ising-type walls in-between them: the magnetic field vanishes during the polarity change rather than rotating as in a Bloch-type wall. For other parameter values $R m_{i}(i=1,2)$, we have also found reversals of Bloch-type. One important observation for the reversals reported here is the correlation with the global energy budget of the flow. The total power $P(t)$ delivered by the motors driving the flow fluctuates in time in a strongly asymmetric manner: the record shows short periods when $P$ is much smaller than its average. They always coincide with large variations in the magnetic field, as shown in figure 5. Either a reversal occurs, or the magnetic field first decays and then grows again with its direction unchanged. Similar sequences, called excursions, are observed in recordings of the Earth's magnetic field. The variation of power consumption during the weakening of the magnetic field is in agreement with the power required to sustain a steady dynamo in the VKS2 experiment (drops by over $20 \%$, that is $20 \mathrm{~kW}$ out of $90 \mathrm{~kW}$ ). However, we 


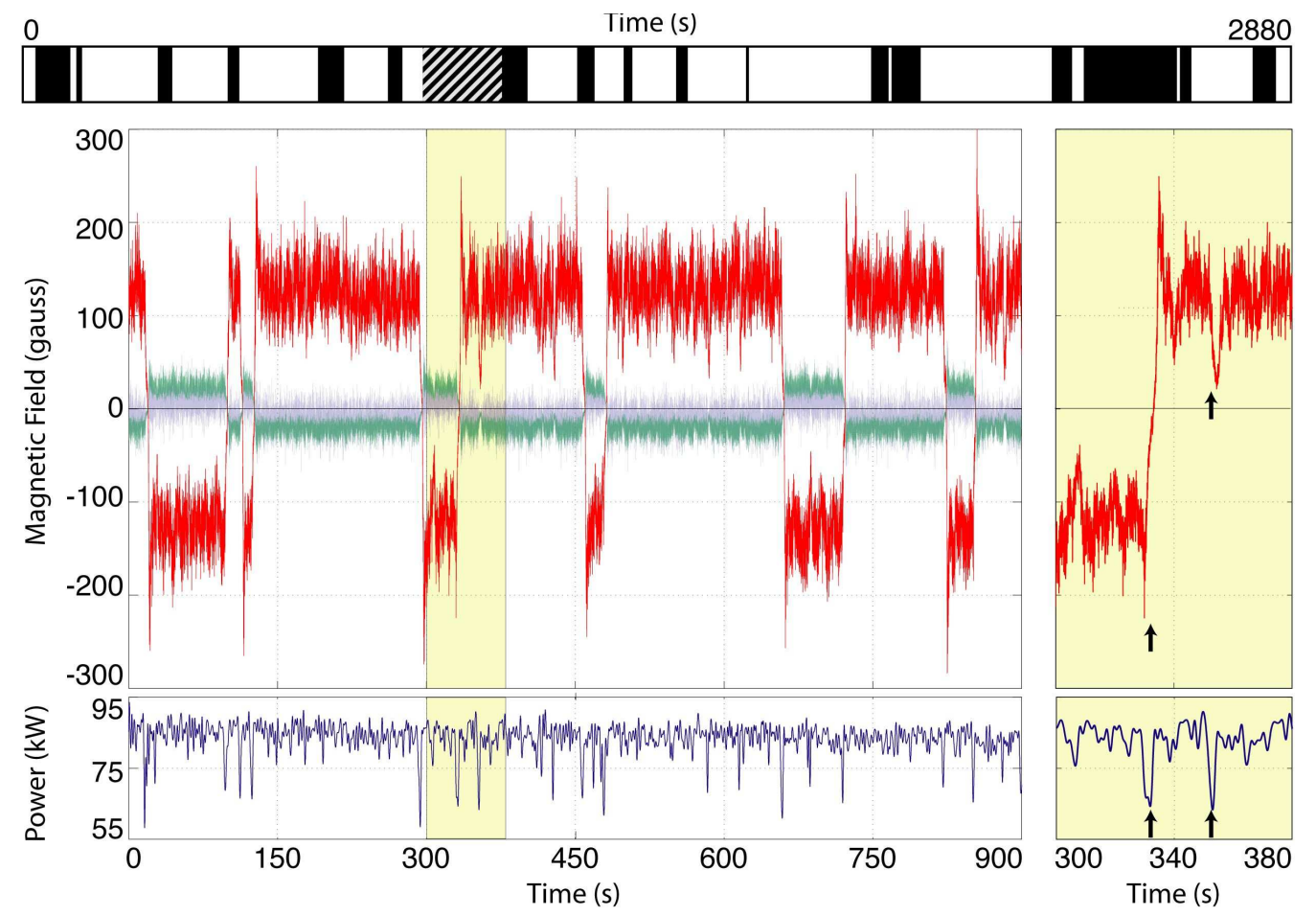

Figure 5. Direct time recordings of the three components of the magnetic field. Disk rotation frequencies, $F_{1}=16 \mathrm{~Hz}, F_{2}=-22: B_{x}$ (blue), $B_{y}$ (red), $B_{z}$ (green). Measurement at point P1 in VKS2h. The upper graph shows the sequences of polarities for the longest in this regime (the shaded area corresponding to the lower plot).

note that in other regions of the parameter space, different regimes also involve changes in polarity without noticeable modification of power.

We have also observed that the trajectories connecting the symmetric states $\mathbf{B}$ and $\mathbf{- B}$ are quite robust despite the strong turbulent fluctuations of the flow. This is displayed in figure 6: the time evolution of reversals from up to down states can be neatly superimposed by shifting the origin of time such that $B(t=0)=0$ for each reversal. Despite the asymmetry due to the Earth magnetic field, down-up reversals can be superimposed in a similar way on up-down ones if $-B$ is plotted instead of $B$. For each reversal the amplitude of the field first decays exponentially. A decay rate of roughly $0.8 \mathrm{~s}^{-1}$ is obtained with a log-lin plot (not shown). After changing polarity, the field amplitude increases linearly and then displays an overshoot before reaching its statistically stationary regime.

Further investigation of this regime will help address from an experimental perspective persistent questions about magnetic field reversals. Some of these concern the role of hydrodynamics and electromagnetic boundary conditions both of them can be experimentally adjusted. Others are related to the dynamics of the magnetic reversals. From inspection of paleomagnetic data, it has been proposed that reversing dynamos and non-reversing ones are metastable 


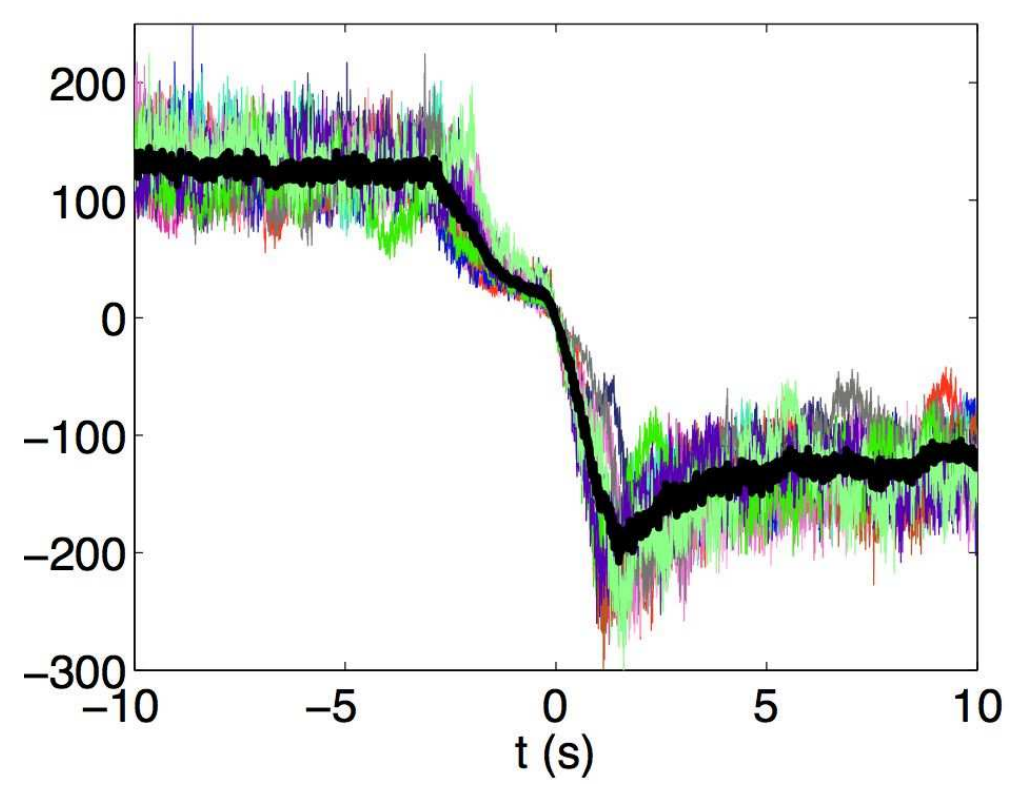

Figure 6. Superimposition of 6 successive reversals from up to down polarity together with 6 successive reversals from down to up polarity with the transformation $\mathbf{B} \rightarrow-\mathbf{B}$. For each of them the origin of time has been shifted such that it corresponds to $\mathbf{B}=0$. The thick black line is the average of all reversals.

states in close proximity [33]. In geodynamo simulations (convective dynamos in rapidly rotating spheres), the flow is often laminar and reversals have been associated to interaction between dipole and higher order modes, with the possibility of reversal precursor events [34]. Field reversals have also been observed in turbulence driven numerical $\alpha^{2}$ and $\alpha-\omega$ dynamos based on mean-field magnetohydrodynamics $[35,42]$. In these, the role of noise was found to be essential, together with the proximity of steady and oscillating states. In many cases, the existence of several dynamo regimes in a narrow region of parameter space has been considered as essential. Our experiment displays this feature: two different stationary dynamo modes bifurcate for $F_{1}=F_{2}$ and respectively $F_{1} \neq F_{2}$ (see section below). Their interaction gives rise to a variety of different dynamical regimes in parameter space. This is a general feature for bifurcations of multiple codimension.

\section{Transitions and chaotic features}

In the VKS experiment, depending on the amount of global rotation, both stationary regimes are observed as well as several secondary bifurcations that lead to more complex dynamics including field reversals - see above. The aim of this section is to discuss these different regimes and to illustrate that they can be understood as the result of a few competing modes in the vicinity of the dynamo threshold. 

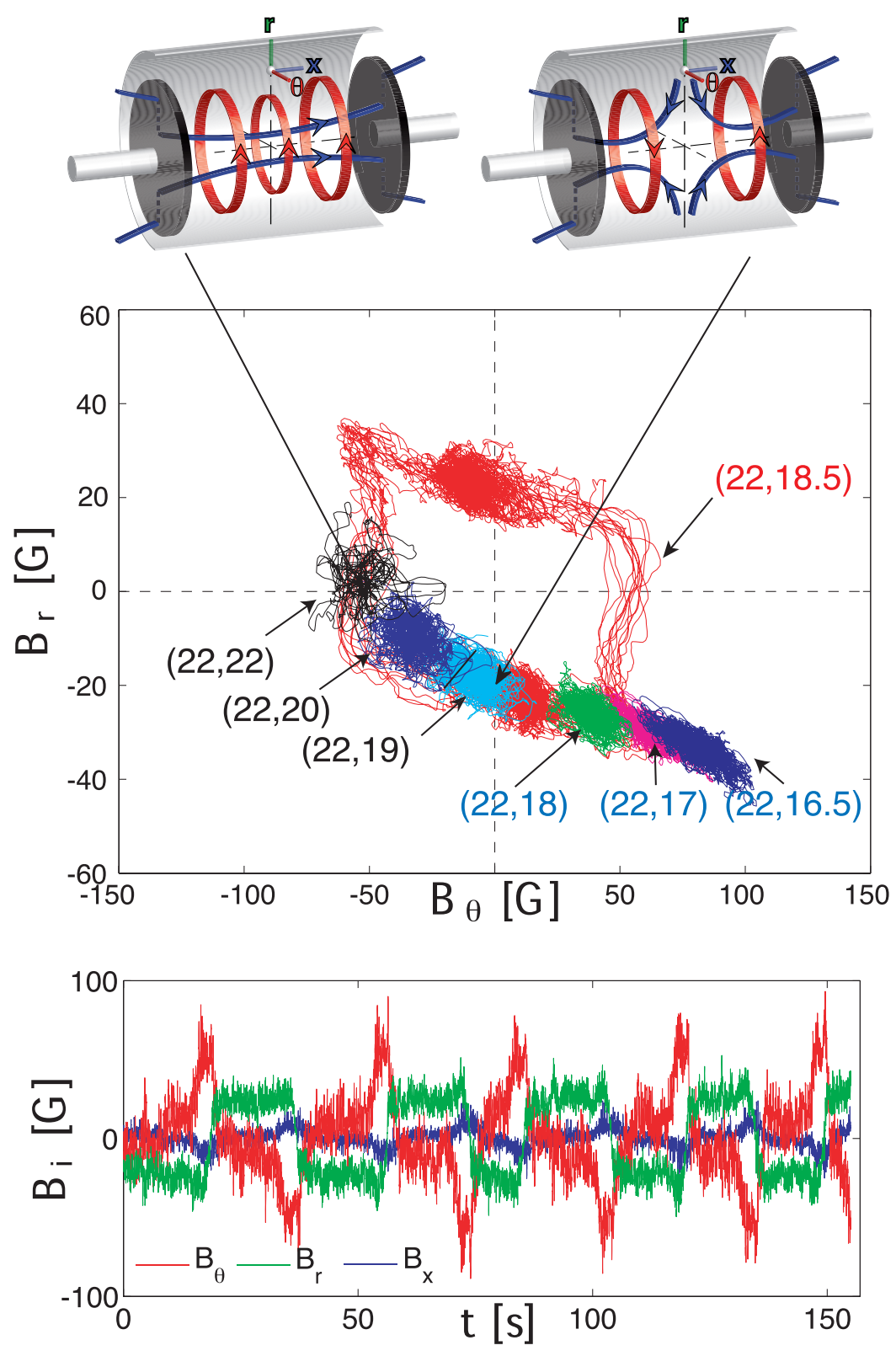

Figure 7. Top: sketch of the axial dipolar (left) and quadrupolar (right) magnetic modes. Middle: location of the different states in the $\left(B_{r}, B_{\theta}\right)$ plane: fixed points corresponding to the stationary regimes for frequencies $\left(F_{1}-F_{2}\right)$; limit cycle observed for impellers counter-rotating at different frequencies $22-18.5 \mathrm{~Hz}$ (red). The magnetic field is time averaged over $1 s$ to remove high frequency fluctuations caused by the turbulent velocity fluctuations. Bottom: time recording of the components of the magnetic field for frequencies $22-18.5 \mathrm{~Hz}$.

Let us consider the case where we start from impellers rotating at $22 \mathrm{~Hz}$, and then the frequency of an impeller, say $F_{2}$, is decreased, $F_{1}$ being kept constant. We first observe a statistically stationary dynamo regime with a dominant azimuthal mean field close to the flow periphery (figure 7, top left). 
This corresponds to the trace labelled $22-22$ in the $\left(B_{r}, B_{\theta}\right)$ plane of figure 7 (middle). As the frequency of the slower impeller is decreased, we obtain other stationary dynamo regimes for which the radial component of the mean field increases and then becomes larger than the azimuthal one (22-20 and 22-19). When we tune the impeller frequencies to 22 and $18.5 \mathrm{~Hz}$ respectively, a global bifurcation to a limit cycle occurs. We observe that the trajectory of this limit cycle goes through the location of the previous fixed points related to the stationary regimes. This transition thus looks like the one of an excitable system: an elementary example of this type of bifurcation is provided by a simple pendulum submitted to a constant torque. As the value of the torque is increased, the stable equilibrium of the pendulum becomes more and more tilted from the vertical and for a critical torque corresponding to the angle $\pi / 2$, the pendulum undergoes a saddle-node bifurcation to a limit cycle that goes through the previous fixed points. Direct time-recordings of the magnetic field, measured at the periphery of the flow in the mid-plane between the two impellers, are displayed in figure 7 (bottom). We propose to ascribe the strong radial component (in green) that switches between $\pm 25 \mathrm{G}$ to a quadrupolar mode (see figure 7, top right)). Its interaction with the dipolar mode (Fig. 2, top left) that is the dominant one for exact counter-rotation, gives rise to the observed relaxation dynamics. This hypothesis is supported by measurements made outside of the equatorial plane $(x=109 \mathrm{~mm}, r=206 \mathrm{~mm})$ where the radial to azimuthal field ratio is much smaller, as it should if the radial field mostly results from the quadrupolar component. We note that it has been often observed that dipolar and quadrupolar dynamo modes can have their respective thresholds in a narrow range of $R_{m}$ [1] and this has been used to model the dynamics of the magnetic fields of the Earth [36] or the Sun [37]. The relaxation oscillation is observed in a rather narrow range of impeller frequency $F_{2}$ (less than $1 \mathrm{~Hz}$ ). When the frequency of the slowest impeller in decreased further, statistically stationary regimes are recovered $(22-18$ to $22-16.5 \mathrm{~Hz}$ in Fig. 2, middle). They also correspond to fixed points located on the trajectory of the limit cycle, except for the case $22-16.5 \mathrm{~Hz}$ that separates from it.

When the rotation frequency of the slowest impeller is decreased further, new dynamical regimes occur. One of them consists in the field reversals described above. The time evolution averaged over the successive reversals (figure 6) can be represented as the trajectory of the system in phase space using a threedimensional plot of $\left[B_{\theta}(t), B_{\theta}(t+\delta t), B_{\theta}(t+2 \delta t)\right]$ (figure 8 , bottom) where $\delta t=1 \mathrm{~s} \sim \tau_{\sigma}$. For each reversal, the field first decays exponentially. This corresponds to the part of the trajectory from $P$ to $Q$ in phase space. The system then leaves $Q$ on a faster time scale and reaches the state with opposite polarity $-P$ after displaying an overshoot in the direct time recording of figure 8 (top). In phase space, this is related to the fact that the trajectory has to circle around the fixed point $-P$ in order to reach it along one of its stable manifold. As proposed by many authors [38], it is tempting to understand these 
field reversals as heteroclinic orbits connecting unstable fixed points in phase space. In the present situation, these fixed points are $P, Q$ together with the symmetric ones $-P$ and $-Q$ obtained by the $\mathbf{B} \rightarrow-\mathbf{B}$ transformation. The trajectory in phase space is amazingly robust despite strong velocity fluctuations. These fluctuations put an upper bound on the duration of phases with a given polarity by preventing the system to stay too close to the unstable fixed points and thus to spend too much time in their vicinity. However, this does not suppress the scale separation between the length of the phases with given polarity and the duration of a reversal. In that sense, turbulent fluctuations have a weak effect on the large scale dynamics of the magnetic field.
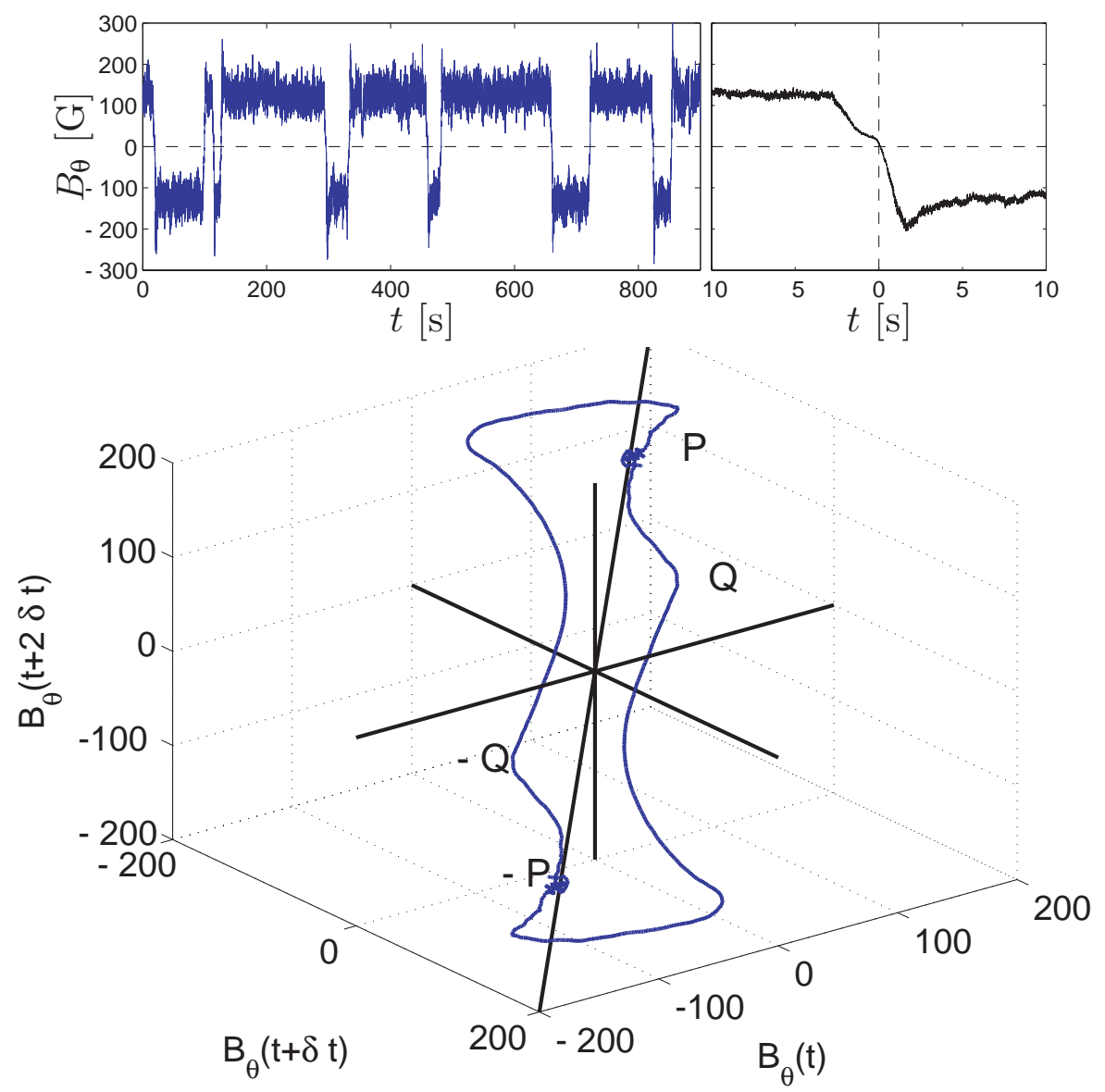

Figure 8. Top: the average of the 12 successive reversals displayed in the direct recording of $B_{\theta}(t)$ (left), is shown on the right figure (see text for explanations). Bottom: plot of the trajectory of the averaged reversal in phase space $\left[B_{\theta}(t), B_{\theta}(t+\delta t), B_{\theta}(t+2 \delta t)\right](\delta t=1 \mathrm{~s})$. Measurements performed at $(x=109 \mathrm{~mm}$, $r=206 \mathrm{~mm}$ ) for impellers rotating at frequencies $22-16 \mathrm{~Hz}$.

We show next that the fixed points $\pm P$ and $\pm Q$ are also involved in other dynamical regimes observed in the vicinity of reversals in the parameter space. For rotation frequencies $22-15 \mathrm{~Hz}$, the magnetic field displays intermittent bursts (figure 9, top left). The most probable value of the azimuthal field is roughly $20 \mathrm{G}$ but bursts up to more than $100 \mathrm{G}$ are observed such that the 
probability density function of the field has an exponential tail (not shown). For rotation frequencies $21-15 \mathrm{~Hz}$, the same type of dynamics occur, but in a symmetric fashion, both positive and negative values of the field being observed (top right). Note also that the magnetic field vanished for a while $(t \sim 300 \mathrm{~s})$. Such extinctions are also observed as transient after changes of the driving in this area of the phase diagram (black $\otimes$ in Fig.1 around $\left(F_{1}, F_{2}\right)=(22,15)$ or $\left.(15,22)\right)$. A section of the phase space is displayed in figure 9 (bottom) by plotting $\left[B_{\theta}(t), B_{\theta}(t+\delta t)\right]$ with $\delta t=1 \mathrm{~s}$ for both regimes of bursts and field reversals. We observe that the bursts occur from the unstable

fixed points $\pm Q$ that were already involved in the trajectories related to field reversals.

\section{Discussion and concluding remarks}

We have collected in this report the main observations made in the VKS experiment, for the configuration detailed in the experimental setup section.

A first finding concerns dynamo generation for the symmetric forcing $F_{1}=F_{2}$. A statistically stationary dynamo grows for $R m$ values in excess of about 32 . The main component has the geometry of an axial dipole in contrast with kinematic dynamo codes taking into account only the mean flow. Cowling's theorem shows that non axisymmetric turbulent fluctuations are essential for the generation of this dynamo. In addition the threshold value is also lower than the kinematic predictions using the mean flow, that are in the range $R_{m}^{c}=40$ to 150 depending on different boundary conditions on the disks and on configurations of the flow behind them $[16,20]$. The generation of the statistically stationary dynamo driven at $F_{1}=F_{2}$ share many characteristic with bifurcations in the presence of noise. For instance threshold values defined using mean field or rms quantities differ. As shown in much simpler experiments, different choices of an order parameter (mean value of the amplitude of the unstable mode or its higher moments, its most probable value, etc) can lead to qualitatively different bifurcation diagrams [39]. This illustrates the ambiguity in the definition of the order parameter for bifurcations in the presence of fluctuations or noise. In the present experiment, fluctuations enter both multiplicatively, because of the turbulent velocity, and additively, due to the interaction of the velocity field with the ambient magnetic field.

A second noteworthy feature is that different dynamical regimes are generated when the flow is driven with impellers counter-rotating at different rates. In addition, these regimes display several characteristic of low dimensional dynamical systems: global bifurcations from fixed points to a relaxation cycle, heteroclinic orbits, chaotic bursts from unstable fixed points. These dynamics can be understood as the ones resulting from the competition between a few 

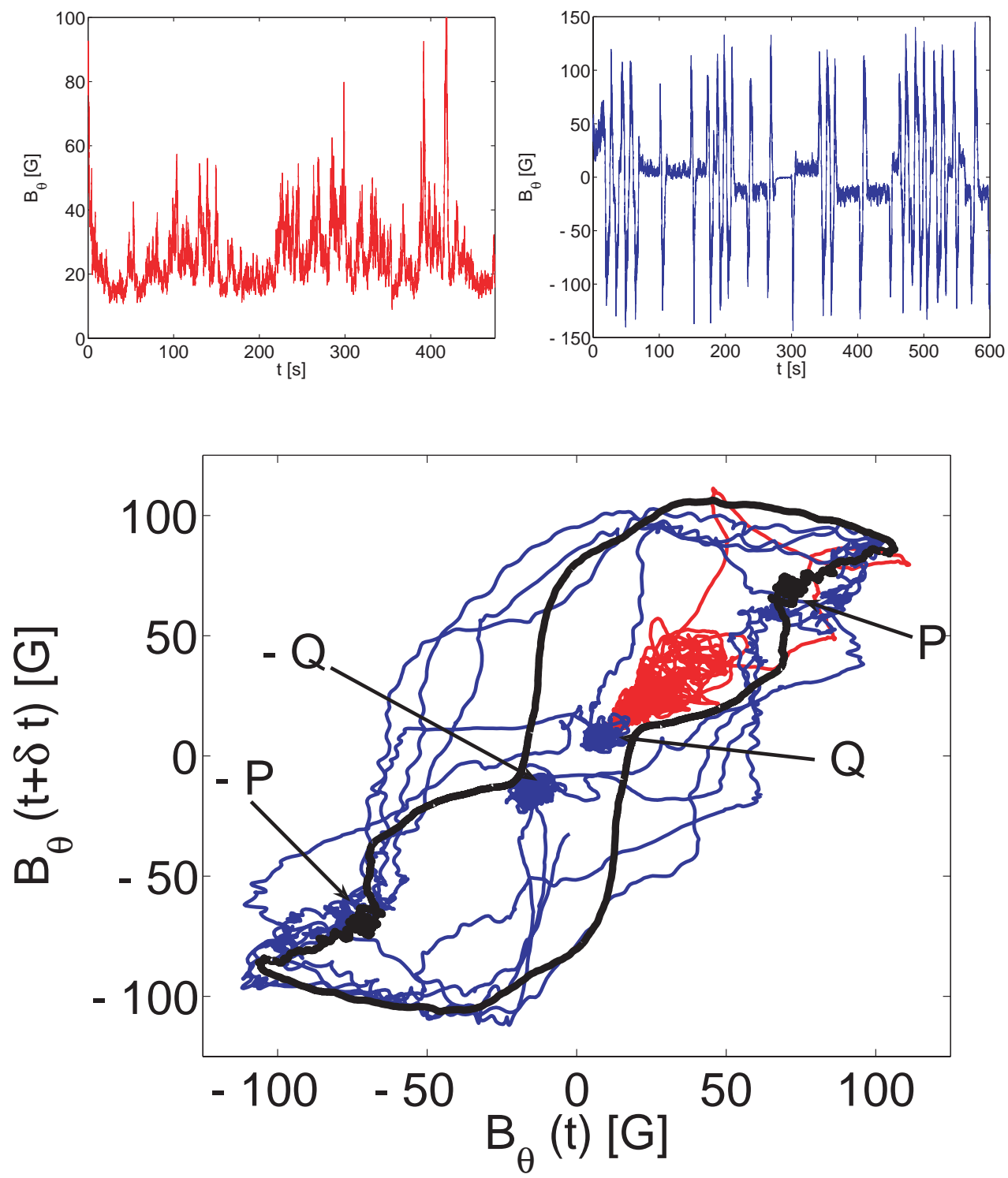

Figure 9. Top: time recordings of the azimuthal component of the magnetic field observed for impellers rotating at (22-15) $\mathrm{Hz}$ (left), (21-15) $\mathrm{Hz}$ (right). Bottom: plot of a cut in phase space $\left[B_{\theta}(t), B_{\theta}(t+\delta t)\right]$ with $\delta t=1 \mathrm{~s}$ for three regimes: in black for field reversals reported in Fig. 3, the magnetic field being rescaled by an ad-hoc factor accounting that the probe location is not in the mid-plane. In blue, for symmetric bursts $(21-15 \mathrm{~Hz})$ and in red for asymmetric bursts $(22-15 \mathrm{~Hz})$. In these last two plots the magnetic field is time averaged over $0.25 \mathrm{~s}$ to remove high frequency fluctuations.

nearly critical modes. This has been observed in other hydrodynamical instabilities since the early experimental studies on codimension-two bifurcations [40]. Competing critical modes have been already considered as phenomenological models of the dynamics of the magnetic fields of the Earth $[36,38]$ or the Sun $[41,37]$ and similar type of dynamics have been obtained. It has been also observed in simulations [34] or mean field models [42] that magnetic field 
reversals can occur when a stationary and an oscillatory modes are in proximity and a simple analytic model has been designed in this spirit [43]. It is also well-known since Rikitake that dynamo models corresponding to a drastic truncation of the governing equations can give rise to complex temporal dynamics [44]. We emphasize that what is remarkable in the present study is the robustness of these low dimensional dynamical features that are not smeared out despite large turbulent fluctuations of the flow that generates the dynamo field. A possible explanation is that the large scale magnetic field is too slow to follow velocity perturbations with time scales comparable to the rotation rate of the impellers or smaller.

\section{Acknowledgements}

We thank M. Moulin, C. Gasquet, J.-B Luciani, A. Skiara, D. Courtiade, J.-F. Point, P. Metz and V. Padilla for their technical assistance. This work is supported by ANR05-0268-03, Direction des Sciences de la Matière et Direction de l'Énergie Nucléaire of CEA, Ministère de la Recherche and CNRS. The experiment is operated at CEA/Cadarache DEN/DTN.

\section{References}

[1] H. K. Moffatt, Magnetic field generation in electrically conducting fluids, Cambridge University Press (Cambridge, 1978)

[2] G. O. Roberts, Phil. Trans. Roy. Soc. London A 271, 411-454 (1972); Yu. B. Ponomarenko, J. Appl. Mech. Tech. Phys. 14, 775-778 (1973)

[3] R. Stieglitz and U. Müller, Phys. Fluids 13, 561 (2001); A. Gailitis et al., Phys. Rev. Lett. 86, 3024 (2001)

[4] F. H. Busse, U. Müller, R. Stieglitz and A. Tilgner, Magnetohydrodynamics 32, 235-248 (1996); K.-H. Rädler, E. Apstein, M. Rheinhardt and M. Schüler, Studia Geophys. Geod. 42, 224-231 (1998); A. Gailitis et al., Magnetohydrodynamics 38, 5-14 (2002)

[5] F. Pétrélis and S. Fauve, Eur. Phys. J. B 22, 273-276 (2001)

[6] A. A. Schekochihin et al., Phys. Rev. Lett. 92054502 (2004); S. Boldyrev and F. Cattaneo Phys. Rev. Lett. 92, 144501 (2004), and references therein.

[7] J.-P. Laval et al., Phys. Rev. Lett. 96204503 (2006)

[8] Y. Ponty et al., Phys. Rev. Lett. 94, 164502 (2005); Y. Ponty et al., New. J. Phys. 9, 296 (2007)

[9] H. C. Nataf et al., GAFD 100, 281 (2006) 
[10] P. Odier, J. F. Pinton and S. Fauve, Phys. Rev. E 58, 7397-7401 (1998); N. L. Peffley, A. B. Cawthorne, and D. P. Lathrop, Phys. Rev. E 61, 5287-5294 (2000); E. J. Spence et al., Phys. Rev. Lett. 96, 055002 (2006); R. Stepanov et al., Phys. Rev. E 73046310 (2006)

[11] M. Bourgoin et al., Phys. Fluids 14, 3046 (2002); F. Pétrélis et al., Phys. Rev. Lett. 90, (17) 174501 (2003); R. Volk et al., Phys. Rev. Lett. 97, 074501 (2006)

[12] R. Monchaux et al., Phys. Rev. Lett. 98, 044502 (2007)

[13] M. Berhanu et al., Europhys. Lett. 77, 59001 (2007)

[14] F. Ravelet et al., Chaotic dynamos generated by a turbulent flow of liquid sodium, submitted to Phys. Rev. Lett. (2008)

[15] L. Marié and F. Daviaud, Phys. Fluids 16, 457 (2004)

[16] F. Ravelet et al., Phys. Fluids 17, 117104 (2005)

[17] F. Ravelet, PhD Thesis, pp. 51, 187-190 (2005) http://tel.archives-ouvertes.fr/tel-00011016/en/

[18] S. Fauve and F. Pétrélis, "The dynamo effect", in "Peyresq Lectures on Nonlinear Phenomena, Vol. II", Ed. J-A Sepulchre, pp. 1-64, World Scientific (2003)

[19] R. Avalos-Zuniga, F. Plunian and A. Gailitis, Phys. Rev. E 68066307 (2003)

[20] F. Stefani et al., Eur. J. Mech. B 25894 (2006)

[21] C. Gissinger et al., Europhys. Lett. 82, 29001 (2008)

[22] D. Sweet et al., Phys. Rev. E 63, 066211 (2001)

[23] S. Aumaître, F. Pétrélis and K. Mallick, Phys. Rev. Lett. 95, 064101 (2005)

[24] F. Pétrélis and S. Aumaître, Eur. Phys. J. B 51357 (2006)

[25] F. J. Lowes and I. Wilkinson, Nature 198, 1158 (1963); 219, 717 (1968)

[26] L. Marié et al., Eur. Phys. J. B 33, 469 (2003)

[27] M. Bourgoin et al., Phys. Fluids 16, 2529 (2004)

[28] F. Pétrélis, N. Mordant and S. Fauve, GAFD 101, 289 (2007)

[29] R. Laguerre, C. Nore, A. Ribeiro, J. Léorat, J.-L. Guermond, F. Plunian, Impact of turbines in the VKS2 dynamo experiment, arXiv:0805.2805

[30] R. Stepanov et al., Phys. Rev. E, 73, 046310 (2006)

[31] R. Volk, PhD thesis, Ecole Normale Supérieure de Lyon (2005), http://tel.archives-ouvertes.fr/tel-00011221/en/

[32] K.-H. Rädler and R. Stepanov, Phys. Rev E 73, 056311 (2006) 
[33] P.L. MacFadden and R.T. Merrill, Physics of the Earth and Planetary Interiors, 91, $253(1995)$

[34] G.R. Sarson and C.A. Jones, Physics of the Earth and Planetary Interiors, 111, 3 (1999) 3; J. Wicht and P. Olson, Geochemistry, Geophysics and Geosystems (G-cubed), 5 (2004)

[35] A. Giesecke, G.R. Rüdiger and D. Elstner, Astron. Nachr. 326, 693 (2005); L. Widrow, Rev. Mod. Phys. 74, 775 (2002)

[36] I. Melbourne, M. R. E. Proctor and A. M. Rucklidge, Dynamo and dynamics, a mathematical challenge, Eds. P. Chossat et al., pp. 363-370, Kluwer Academic Publishers (2001)

[37] E. Knobloch and A. S. Landsberg, Mon. Not. R. Astron. Soc. 278, 294 (1996)

[38] P. Chossat and D. Armbruster, Proc. Roy. Soc. London A 459, 577 (2003) and references therein.

[39] R. Berthet et al., Physica D 174, 84-99 (2003)

[40] S. Fauve et al., Phys. Rev. Lett. 55, 208 (1985); R. W. Walden et al., Phys. Rev. Lett. 55, 496 (1985); I. Rehberg et al., Phys. Rev. Lett. 55, 500 (1985)

[41] S. M. Tobias, N. O. Weiss and V. Kirk, Mon. Not. R. Astron. Soc. 273, 1150 (1995)

[42] F. Stefani and G. Gerbeth, Phys. Rev. Lett. 94, 184506 (2005)

[43] P. Hoyng and J. J. Duistermaat, Europhys. Lett. 68, 177 (2004)

[44] T. Rikitake, Proc. Camb. Phil. Soc. 54, 89 (1958); D. W. Allan, Proc. Camb. Philos. Soc. 58, 671 (1962); A. E. Cook and P. H. Roberts, Proc. Camb. Philos. Soc. 68, 547 (1970); W. V. R. Malkus, EOS, Trans. Am. Geophys. Union 53, 617 (1972); P. Nozières, Phys. Earth Planet Inter., 17, 55 (1978) 